\title{
Development of a Tensile Testing Apparatus for Human Hair
}

\author{
Kento Fujita* and Hironori Tohmyoh \\ Department of Finemechanics, Tohoku University, Aoba 6-6-1, Aramaki, Aoba-ku, Sendai, Japan \\ *fujita@ism.mech.tohoku.ac.jp
}

Introduction. Because human hair has been found to hold various information on our health, such as disease, aging $^{(1)}$, etc., investigating various properties of hair has attracted considerable attentions recently ${ }^{(2,3)}$. Human hair has multi-layered structure, and therefore, mechanical properties of the human hair might be important parameter for the abovementioned purpose in the future. Among various mechanical properties, the tensile test is one of the suitable way to get useful information from a hair because it can be performed under various tensile rate.

In this research, we developed a tensile testing apparatus and scheme for evaluating a human hair. Young's modulus and tensile stress of a human hair were measured under various tensile rate.

Materials and Methods. Figure 1(a) shows the whole view of the developed testing apparatus, and it is consisted of a motorized stage, a load cell and a pair of sample grippers. Before testing, geometry of the crosssection of the hair to be tested was examined with a microscope. Firstly, we considered the way to grip the ends of the hair sample, and a method to use a paper was adapted $^{(4)}$. Two pieces of paper with rectangle hole were prepared, and a hair were inserted between them. The hair sample was then held by two sample grippers, see Fig. 1(b). After holding the hair sample on the sample grippers, both sides of the papers were cut so that tensile load is directly applied to the hair sample. The gauge length was fixed at $10 \mathrm{~mm}$ for all the cases. The loaddisplacement curves for the hair samples were recorded under the tensile rates of 1,10 and $1000 \times 10^{-6} \mathrm{~m} \mathrm{~s}^{-1}$ at room temperature. Young's modulus and tensile stress were determined from 5 samples at each tensile rate.

Results and Discussion. Figure 2 shows an example of the load-displacement curve for the hair sample obtained at the tensile rate of $1 \times 10^{-6} \mathrm{~m} \mathrm{~s}^{-1}$. Initially, the load increased linearly against the displacement, and the elastic region was confirmed. After the hair sample was yielded, behavior of the load-displacement curve was found to be divided by the yield and the post-yield regions ${ }^{(3)}$.
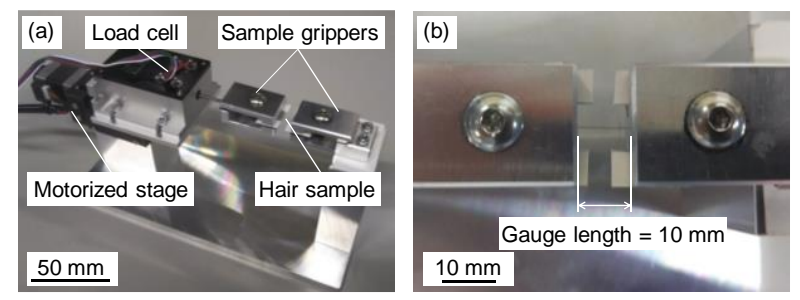

Fig. 1 (a) Whole view of the tensile testing apparatus. (b) Details of the sample grippers and hair sample.

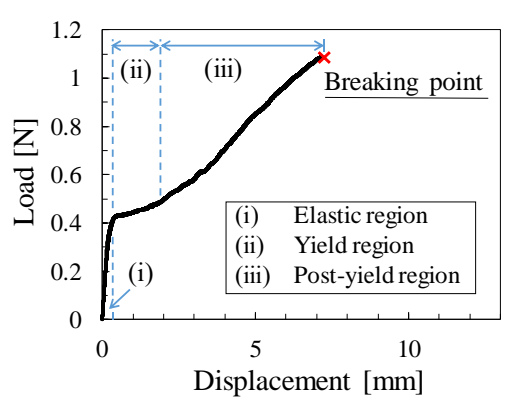

Fig. 2 An example of the load-displacement curve of a human hair sample obtained at $1 \times 10^{-6} \mathrm{~m} \mathrm{~s}^{-1}$.
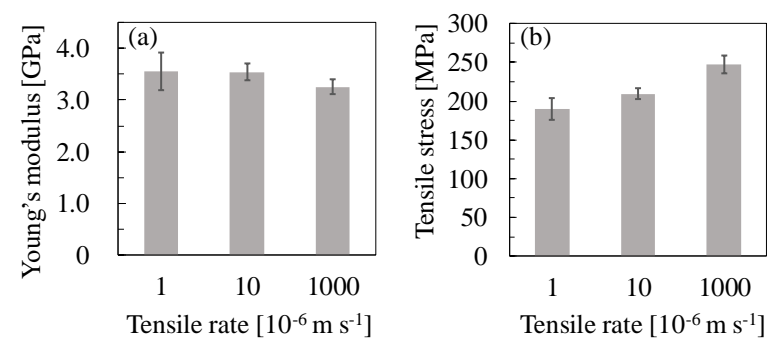

Fig. 3 (a) Young's modulus and (b) tensile stress obtained at various tensile rate.

Figure 3(a) displays the Young's modulus of the hair sample determined at each tensile rate, and it was found to be independent with the tensile rate. The tensile stress was shown in Fig. 3(b). Here the tensile stress was determined by dividing the breaking load by the crosssectional area of the hair. The tensile stress of the hair sample obtained at the tensile rate of $1000 \times 10^{-6} \mathrm{~m} \mathrm{~s}^{-1}$ became greater than that for others, although Young's modulus was independent with the tensile rate. This suggests that the effect of the tensile rate on fracture characteristics might be ignored at the lower tensile rate.

Conclusion. In this research, we developed a tensile testing apparatus for a single human hair, and conducted a tensile test to evaluate the tensile properties of a hair.

Acknowledgement. This work was supported by JSPS KAKENHI Grant No. 18H01331.

\section{References.}

(1) Sayahi, E., Harizi, T., Msahli, S., and Sakli, F., Int. J. Cosmet. Sci., 38, (2016), 470-475.

(2) Tohmyoh, H., Ishihara, M., Akanda, M. A. S., Yamaki, S., Watanabe, T., and Iwabuchi, T., J. Biomech., 44, (2011), 2833-2837.

(3) Yu, Y., Yang, W., Wang, B., and Meyers, M. A., Mater. Sci. Eng. C, 73, (2017), 152-163.

(4) Thozhur, S. M., Crocombe, A. D., and Smith, P. A., J. Mater. Sci., 41, (2006), 1109-1121. 